Des Allemands de la Volga et la lutte contre l'analphabétisme en URSS dans les années 1910-1930

\title{
Ekaterina Alexeeva
}

\section{(2) OpenEdition Journals}

Édition électronique

URL : http://journals.openedition.org/esp/513

DOI : 10.4000/esp.513

ISSN : 2532-0319

Éditeur

Centre d'Information sur l'Éducation Bilingue et Plurilingue

\section{Édition imprimée}

Date de publication : 1 juin 2015

Pagination : 7-13

ISSN : 1127-266X

Référence électronique

Ekaterina Alexeeva, «Des Allemands de la Volga et la lutte contre l'analphabétisme en URSS dans les années 1910-1930 », Éducation et sociétés plurilingues [En ligne], 38 | 2015, mis en ligne le 01 mars 2016, consulté le 25 février 2021. URL : http://journals.openedition.org/esp/513 ; DOI : https://doi.org/ 10.4000/esp.513 


\section{DES ALLEMANDS DE LA VOLGA ET LA LUTTE CONTRE L'ANALPHABÉTISME EN URSS DANS LES ANNÉES 1910-1930(1)}

\section{Ekaterina AlexeEva}

Quest'articolo è dedicato alle specificità dell'adattamento da parte dè Tedeschi del Volga alla vita russa negli anni 19101930. Studieremo alcuni fattori che hanno condizionato il bilinguismo in questa repubblica sovietica tedesca in quest'epoca storica (per esempio la lotta contro l'analfabetismo e la costruzione di scuole a base russa nelle città e nei villaggi). Nella nostra ricerca ci baseremo su fonti scritte che hanno mantenuto le tracce dei provvedimenti linguistici applicati dal potere sovietico alla popolazione dei cantoni tedeschi del Volga.

This article shows how Volga Germans adapted to Russian life during the 1910-1930s. Certain factors were instrumental in developing bilingualism in the Volga German Autonomous Soviet Socialist Republic during this period of history, as for instance the struggle against illiteracy and the opening of Russian-based schools in German-speaking towns and villages. Our research is based on written sources in which the linguistic measures applied by the USSR to the population of the German-speaking counties of the Volga are still visible.

Key-words: Germans of the Volga, USSR, 1910-1930, illiteracy, cultural and linguistic integration

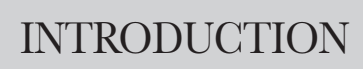

$\mathrm{C}$

et article est consacré au bilinguisme russo-allemand dans la communauté allemande de la Volga dans les années 1910-1930. Les spécificités de l'adaptation des Allemands à la vie russe et leur intégration sont marquées par de nombreux facteurs historiques et sociaux. L'histoire des Allemands de la Volga commence en 1763 avec le manifeste de la tsarine russe d'origine allemande Catherine II, qui encourageait l'installation de colons allemands en Russie.

$\mathrm{Au}$ début du $\mathrm{XX}^{\mathrm{e}}$ siècle on comptait 190 colonies allemandes avec 405,500 personnes de nationalité allemande. En 1918 la communauté allemande obtient le statut de République Socialiste Soviétique Autonome des Allemands de la Volga, en allemand die Autonome Sozialistische Sowjet-Republik der WolgaDeutschen, ou ASSRdWD. Selon le recensement de 1920, la communauté allemande de la Volga comptait 442,362 personnes. En 1937, l'ASSRdWD était peuplée par 322,652 Allemands et 106,466 Russes. Les Allemands représentaient une communauté qui participait activement à la vie de l'URSS.

Malgré les difficultés quotidiennes, les Allemands préservèrent soigneusement leur patrimoine linguistique et culturel en s'inté- 
Des Allemands de la Volga et la lutte contre l'analphabétisme en urss dans les années 1910-1930

E. Alexeeva

LA LUTTE CONTRE L'ANALPHABÉTISME grant à la vie russe. Ils ne restèrent pas «une colonie isolée», fermée au monde qui évoluait autour d'elle. Au contraire, en renforçant leur engagement dans la vie du groupe, ils allaient, mieux qu'aucun autre peuple de Russie, savoir utiliser les voies de la politique pour défendre leurs intérêts. Au milieu des transformations qui allaient secouer la Russie, ils allaient prendre parti et dès que les structures administratives leur en donneraient l'opportunité, ils sauraient affirmer leur identité d'une manière constructive (Guillemard 1999).

En évoquant les spécificités du bilinguisme dans la communauté allemande de la Volga dans les années 1910-1930, il faut prendre en considération la population des villages qui était née avant la Révolution de 1917. C'étaient des communautés qui maintenaient les traditions culturelles et linguistiques de leurs ancêtres. Leur population était assez homogène et monolingue (en allemand). De nombreux cas de bilinguisme y étaient recensés néanmoins. C'étaient des bilingues avec l'allemand en emploi actif et le russe en emploi passif.

A cette époque, la propagande soviétique pénétrait tous les domaines de la vie des Allemands de la Volga. De nombreuses sources écrites de cette période historique (journaux, revues, livres) ont gardé les traces des mesures linguistiques appliquées par le pouvoir soviétique à la population dans les cantons allemands de la Volga.

On peut noter un intérêt élevé des Allemands de la Volga pour l'édition. En 1937, on comptait dans l'ASSRdWD vingt-et-un journaux et revues allemands, dont 16 étaient édités en allemand et 5 en russe. En voici quelques exemples: Trudovaja pravda [La vérité du travail], Leninskij put' [La voie de Lénine], Nachrichten, Bolschewik, Der Kampf, Der Kolonist, Kollektivist, Kommunist, Lenins Weg, Rot Front, Rote Fahne, Rote Jugend, Stalinist, Stalinez, Stalins Weg, Zum Kommunismus. Ces sources écrites nous aident à restaurer le tableau de la vie de cette minorité nationale à cette époque et à mesurer le rôle des deux langues, l'allemand et le russe, dans sa vie quotidienne.

La révolution bolchevique de 1917 influença considérablement la vitalité ethnolinguistique des Allemands de la Volga du point de vue sociolinguistique. Ils se trouvèrent involontairement engagés dans toutes les activités publiques qui nécessitaient une bonne connaissance du russe et favorisaient le développement du bilinguisme.

Les années 1910-1930 furent marquées par la lutte contre l'anal- 
Des Allemands de la Volga et la lutte contre l'analphabétisme en urss dans les années 1910-1930

E. Alexeeva phabétisme dans l'Union soviétique. Les forces de la jeune république soviétique allemande se sont concentrées dans ce domaine. Ces mesures ont touché la population de tous les coins éloignés des cantons (Les cantons étaient des unités administratives dans l'ASSRdWD entre 1920-1941): villages, campagnes, kolkhozes (coopératives agricoles en Union soviétique). Voilà comment est décrite cette situation dans un des journaux des Allemands de la Volga:

«L'une des mesures importantes visant à élever le niveau d'instruction des kolkhoziens est l'édification de 23 écoles pour adultes, pour 609 kolkhoziens. La tâche de ces écoles consiste à donner une instruction du niveau de la quatrième année primaire. Aussi, il est nécessaire d'organiser 13 écoles de niveau supérieur pour 330 auditeurs. La tâche essentielle consiste à donner une instruction jusqu'à la sixième année secondaire» (Leninskij put', 1936, références de page et de numéro inconnues).

Comme les populations des cantons allemands étaient mélangées, les pouvoirs locaux appliquaient aux russophones et aux germanophones les mêmes mesures «culturelles». Des centaines de villages allemands et russes furent impliqués dans la lutte contre l'analphabétisme. Curieusement, l'enseignement se passait essentiellement en russe.

«Le canton de Pokrovsk (capitale de la république allemande) compte neuf écoles (une école par village). On compte 1,357 écoliers et 27 professeurs. Il y a un professeur pour 52 écoliers. Toutes les écoles sont financées par l'Etat. Toutes les études se déroulent en russe. Il existe deux bibliothèques et deux ' $i z b a$ Čital'nja' (2): 1,869 personnes se sont inscrites aux cours de liquidation de l'analphabétisme. [...] Les cours ont eu lieu à Uzmorie, General'skoe, Kvasnikov (villages du canton de Pokrovsk), il y a tous les manuels nécessaires» (Trudovaja pravda, 1923).

Si dans la ville centrale de la république allemande il y avait au moins une école allemande sur onze écoles russes, ce qui permettait à la population allemande de maintenir ses connaissances de la langue maternelle, dans les villages et campagnes germanophones la situation était plus complexe. Les articles de presse de cette époque gardent de nombreux témoignages sur des tentatives entreprises par des gens simples pour attirer l'attention des pouvoirs locaux sur la langue maternelle des Allemands de la Volga. Cela concernait surtout les habitants des villages dont le niveau de connaissance du russe était assez bas. Dans leurs lettres, les villageois et les kolkhoziens allemands déploraient le manque de littérature en langue allemande: 
Des Allemands de la Volga et la lutte contre l'analphabétisme en urss dans les années 1910-1930

E. Alexeeva
«Il a été remarqué que certains dirigeants des organisations cantonales ont troublé la politique nationale selon la Constitution de Staline. Par exemple, le Kantzemupravlenïe'(3), la Baltzerskaja MTS (4), le Kantpotrebsojuz (5) envoient les ordres dans les villages allemands en langue russe» (Leninskij put', 1937).

Afin d'améliorer la lutte contre l'analphabétisme, on organise toutes sortes d'activités (séances de cinéma, randonnées à ski, cercles de tireurs), dont la principale est l'«izba-čital'nja» (2). Dans un journal datant de 1936, il est question du «projet culturel des kolkhoziens pour la période d'hiver». Le but essentiel de ce projet est de «liquider l'analphabétisme dans le canton pour le $1^{\mathrm{er}}$ avril 1937 au plus tard» (Leninskij put', 1936).

La lecture à haute voix des textes politiques est l'une des mesures de la propagande politique de cette époque. Pour améliorer le niveau de culture politique des Allemands, des cours de lecture des discours de Staline sont organisés dans tous les cantons. Un journal cantonal écrit, par exemple, qu'il faut «organiser des conférences politiques, par exemple, sur le discours du camarade Staline au VIII ${ }^{\text {eme }}$ Congrès des Soviets de l'Union soviétique, les résultats du Congrès des Allemands de la Volga de la région de Saratov, la situation politique en Espagne, les nouvelles internationales [...]» (Leninskij put', 1936).

D’après les journaux locaux, les paysans et les kolkhoziens manquaient souvent ces lectures. L'une des causes était une mauvaise connaissance de la langue russe. Un article du journal Leninskij put' décrit ce problème comme suit:

«Au kolkhoze Šilling (Schilling) de Vorošilov on ne prête pas suffisamment attention à l'étude de la Constitution de Staline. On a organisé un cercle kolkhozien pour l'étude de la Constitution, qui aurait dû se réunir trois fois par semaine. Mais ses activités ont cessé. Depuis le mois de décembre de l'année passée, un seul cours a eu lieu» (Leninskij put', 1937).

Afin d'élever le niveau culturel des habitants de la république, on ouvrit des écoles publiques spéciales. C'est l'exemple de l'«Obsoupartškola» [Ecole générale soviétique du Parti]. Dans ces écoles les gens peuvent perfectionner leurs connaissances dans les domaines suivants: agriculture, mécanique, commerce. Les Allemands participent activement à ces études. On lit dans le journal Trudovaja pravda:

«Grâce au travail préparatoire, la sélection à l'école s'est bien passée. Au cours d'une semaine, 180 personnes de tous les cantons ont été sélectionnées. [...] 40 personnes sur 180 n'ont pas pu être admises, généralement à cause de connaissances insuffisantes. 
Des Allemands de la Volga et la lutte contre l'analphabétisme en urss dans les années 1910-1930

E. Alexeeva
Ainsi, il reste 35 places [...]. Actuellement, l'école compte 160 étudiants, dont 98 sont au premier niveau (30 personnes au département allemand et 68 au département russe) et 71 personnes au deuxième niveau d'étude (26 en allemand et 45 en russe). D'après la nationalité, les étudiants se répartissent de la manière suivante: Allemands 40\%, Russes 60\%. Les épreuves d'entrée permettent de juger du niveau de préparation satisfaisant des étudiants. Les cours se passent bien» (Trudovaja pravda, 1923, n 90, p. 8).

La popularisation des valeurs soviétiques s'exprima à travers les fêtes dans les écoles des villages où les populations russophone et germanophone étaient mélangées. Dans un des numéros de 1937 on lit l'article intitulé Notre Pouchkine:

«Le 13 février, 150 kolkhoziens se sont réunis au club du village Moor pour fêter la centenaire de la mort du génial poète russe Pouchkine. Le directeur d'école le camarade Welzemeier a présenté un exposé sur la vie et l'œuvre de Pouchkine. Après l'exposé, les écoliers ont déclamé des poèmes de Pouchkine traduits en allemand 'Derevni' ['Les campagnes'], 'K Čaadaevu' ['Missive à Čaadaev'], 'Poslanie v Sibir" ['Missive en Sibérie']; 'Osen" ['L'Automne']» (Leninskij put', 1937, nº 41, p. 1).

La lutte contre l'analphabétisme et l'édification d'écoles à base russe ne sont pas les seules mesures favorisant le bilinguisme dans les cantons allemands. La propagande antireligieuse occupe une place centrale dans la formation de l'idéologie soviétique. Paradoxalement, Noël était fêté dans les villages germanophones de l'Union soviétique. Mais cette fête était en réalité consacrée à une propagande antireligieuse.

«Les résultats du Noël des komsomols à Pokrovsk. Le 24 décembre au club Prolétaire ont été faits de nombreux exposés, spectacles et mises en scène antireligieux. Avant les spectacles ont été faits les exposés suivants: 'L'essence et la signification du Noël chrétien' et 'L'origine de Noël et des rites chrétiens'. Il y a eu beaucoup de jeunes, d'adultes et de paysans dans tous les clubs [...]. Les résultats de la propagande antireligieuse sont satisfaisants» (Trudovaja pravda, 1923).

Dans le même journal nous trouvons un autre article consacré à ce sujet:

«Le Noël des komsomols à Golyj Karamyš (village dans la région de Saratov) La fête de Noël à Golyj Karamyš a été un succès. [...] La veille de Noël, les komsomols ont mis en scène la pièce allemande révolutionnaire Ugnetennye ['Les opprimés']. Les enfants et les pionniers ont mis en scène la pièce russe Strannik ['Le pèlerin']. Avant la pièce, le secrétaire du KK RKP (comité cantonal du 
Des Allemands de la Volga et la lutte contre l'analphabétisme en urss dans les années 1910-1930

E. Alexeeva

\section{CONCLUSION}

\section{RÉFÉRENCES BIBLIOGRAPHIQUES}

parti communiste russe) a fait l'exposé 'L'essence et la signification du Noël chrétien'. Le public a été content. Puis on a dansé. Cette soirée nous a permis de faire un pas en avant dans la lutte antireligieuse» (Trudovaja pravda, 1923).

Le bilinguisme russo-allemand sur le territoire de la communauté allemande dans les années 1910-1930 était conditionné par de nombreux facteurs historiques, sociaux, politiques. La lutte contre l'analphabétisme, par exemple, dans la république allemande de la Volga fut décisive pour la vitalité de la langue allemande sur le territoire de l'Union soviétique. Cette lutte a rempli le rôle de catalyseur dans le processus de l'implantation de la langue russe dans la communauté allemande. L'édification d'écoles à base russe dans les villes et les villages de la république allemande dans les années 1930 a aussi joué un rôle important dans ces processus. En même temps, nous observons une assez forte tendance des Allemands de la Volga à préserver leur patrimoine langagier et culturel, ce qui s'exprima, par exemple, dans l'apparition d'imprimeries et de nombreuses maisons d'édition vers le début du $\mathrm{XX}^{\mathrm{e}}$ siècle. Nous pouvons juger de l'intégration active des Allemands aux valeurs soviétiques et à la langue russe. Cela a duré jusqu'à 1941, l'année de leur déportation en Sibérie et en Asie Centrale.

GUILLEMARD G. 1999. L'histoire des Allemands de la Volga. 17641999. Mémoire pour le Diplôme d'Études approfondies Cultures et Sociétés. Université du Havre. http://www.alternativen.homepage.t-online.de/histvolgal.html

Leninskij put': Organ Balcerskogo Kantkoma i Kantispolkoma Nemrespubliki [La voie de Lénine: hebdomadaire du comité cantonal de Balzer et du comité exécutif de canton de la République des Allemands de la Volga], 1937, Balzer.

Trudovaja pravda: Ežednevnyj organ Avtonomnï Socialističeskoj Respubliki Nemcev Povolžja [La vérité du travail: quotidien de la République autonome socialiste des Allemands de la Volga], 1923, Pokrovsk. 
Des Allemands de la Volga et la lutte contre

l'analphabétisme en urss dans

les années 1910-1930

\section{E. Alexeeva}

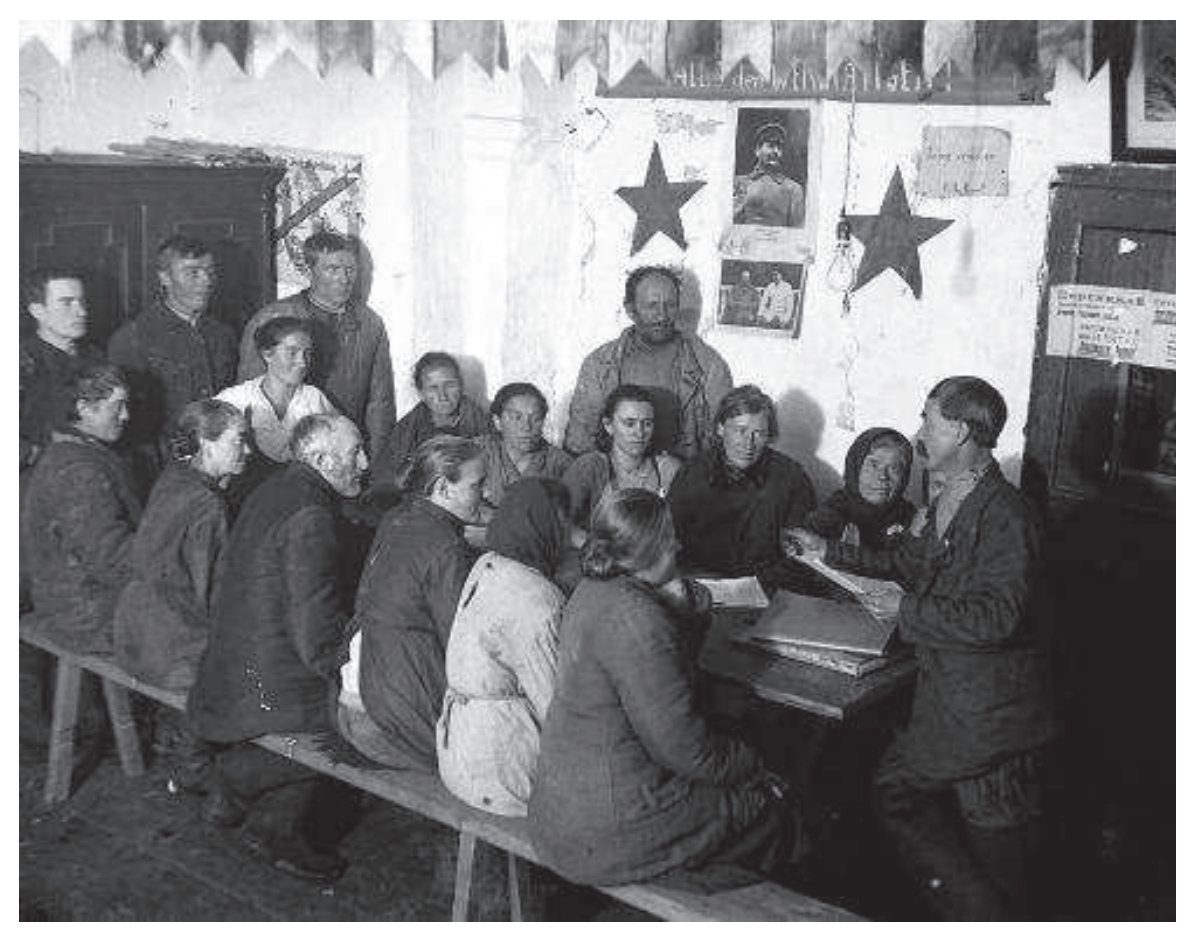

Kolkhoziens allemands en réunion de travail (1933)

http://humus.dreamwidth.org/5472041.html

\section{Notes}

(1) Cet article est paru en 2013 sous le titre Contacts de langues dans la communauté allemande de la Volga dans les années 1910-1930, Univ. de Lausanne, Cahiers de l'ILSL, nº 35: 45-59.

(2) «Izba-Čital'nja» est une maison dans le village destinée à la lecture des textes de la propagande politique pour la population qui ne savait pas lire et écrire. Les izba-Čital'nja étaient populaires dans les années 1920-1930.

(3) Kantonal'noe zemel'noe upravlenie - direction immobilière du canton

(4) Balcerskaja meždugorodnaja telefonnaja stancïa - service téléphonique interurbain

(5) Kantonal'nyj potrebitel'skï sojuz - société cantonale des consommateurs 